\title{
Alcune copie dell'“Almanach" del 1535 di François Rabelais
}

\section{Alessandro Vitale Brovarone}

\section{(2) OpenEdition}

1 Journals

\section{Edizione digitale}

URL: http://journals.openedition.org/studifrancesi/2034

DOI: 10.4000/studifrancesi.2034

ISSN: 2421-5856

\section{Editore}

Rosenberg \& Sellier

\section{Edizione cartacea}

Data di pubblicazione: 1 aprile 2014

Paginazione: 65-74

ISSN: 0039-2944

\section{Notizia bibliografica digitale}

Alessandro Vitale Brovarone, "Alcune copie dell"'Almanach" del 1535 di François Rabelais », Studi

Francesi [Online], 172 (LVIII | I) | 2014, online dal 01 avril 2014, consultato il 18 septembre 2020. URL : http://journals.openedition.org/studifrancesi/2034 ; DOI : https://doi.org/10.4000/studifrancesi.2034

\section{(c)}

Studi Francesi è distribuita con Licenza Creative Commons Attribuzione - Non commerciale - Non opere derivate 4.0 Internazionale. 


\title{
TESTI INEDITI E DOCUMENTI RARI
}

\section{Alcune copie dell"Almanach" del 1535 di François Rabelais}

\begin{abstract}
An investigation on the ancient book collection of the Seminar of Alessandria, survived to the flood of 1994, led to the discovery of a series of copies of the 1535 Almanach of Rabelais. The existence of the text was already known, but just a couple of small fragments had previously been discovered, in Paris. Now we have the entire text, in many copies, each of which slightly incomplete. The copies of the Almanach were used as flyleaves for the volumes of an edition of Giason del Maino's Commentaries on the Corpus Iuris. An accurate work of physical and virtual restoration, and the study of the position of the folios in the volumes of the Commentaries, can help to see some connections between the Almanach, the Commentaries and other works printed by Gryphius and Juste in Lyon, and the total lack of interest shown by the Italian owners of the volumes (probably students of the Faculty of Law in Pavia) towards the Almanach, even if Rabelais' printed signature was extremely evident.
\end{abstract}

Le circostanze particolari di un ritrovamento possono dare indicazioni su possibili linee di ricerca, creando anche aspettative future. Ciascun ritrovamento di dati o di fonti spesso sfugge ad una vera metodica di ricerca, e vede quasi sistematicamente negata ogni forma di proporzionalità fra la fatica e il risultato, salvo che in un bilancio molto generale, che è poi quello che davvero interessa. In più, il ritrovamento sarà ritenuto più o meno fortunato indipendetemente dalle capacità e dalla costanza del ritrovatore. Quest' ultima però ha un peso non indifferente.

La specifica fortuna di trovare un inedito di François Rabelais, poco meno che autografo, non è di tutti i giorni; diventa meno peregrina se si verifica all'interno di una pratica di lavoro improntata alla convinzione che sia ben poco fondata la consolidata opinione che tutto quel che è importante sapere lo si sappia già.

Nella ricerca attenta indirizzata verso materiali che la storia ci trasmette quasi controvoglia ho avuto come compagni diversi allievi, che ho costantemente indirizzati fuori dalle strade principali, troppo affollate e troppo percorse. Nel caso specifico, due allieve, Sabrina Lamparelli e Erika Racca, indirizzate verso lo studio e il censimento di edizioni del XVI secolo, si erano trovate a svolgere, con altri giovani catalogatori, il lavoro difficile di riordino del fondo antico della Biblioteca del Seminario di Alessandria, devastato dall'alluvione del 1994.

Le alluvioni hanno effetti dirompenti sulla conservazione: la carta assorbe facilmente l'acqua, si gonfia, e di conseguenza le legature si aprono: non solo i fili di cucitura, ma anche i cartoni dei piatti diventano evidenti. Poi molto spesso le muffe invadono la carta e la sfaldano: quest'ultimo dato comporta problemi non piccoli di politica della conservazione. Il primo dato è che quando c'è un'alluvione per forza di cose non si tratta di un fenomeno ristretto e puntuale; raramente resta colpita una sola biblioteca. Di conseguenza il problema principale, in caso di alluvione, non è tanto l'esistenza di metodi di intervento e trattamento dei materiali, quanto la disponibilità della forza e delle circostanze adatte per un intervento su larga scala. Ad esempio i 
procedimenti per congelamento, che prevengono danni ulteriori, raramente dispongono di volumetrie sufficienti per eventi devastanti su ampie aree. Presumibilmente la collocazione delle biblioteche in luoghi più elevati e più sicuri sarebbe una soluzione non disprezzabile: ma è molto spesso contraddetta dalle scelte di fatto; basti pensare a quante biblioteche più o meno recenti hanno una bella e incongrua vista sul fiume.

Ma d'altra parte gli eventi dirompenti possono comportare conseguenze collaterali non del tutto negative. Ad esempio aprendo, come si è detto, le legature. $\mathrm{Fu}$ così che un giorno, forse nel 1996, le mie due allieve mi chiamarono al telefono, dicendomi di aver trovato una cosa strana, fogli stampati in due colori, un po' in su e un po' in giù. Ho in quel momento cercato di spiegare come una pratica, detta imposizione, poteva spiegare quella situazione. Non ci fu il tempo di finire la frase, interrotta da un silenzio, e poi: «Cosa sia l'imposizione lo sappiamo benissimo: qui il caso è complicato».

Non è spiacevole sentirsi inutili quando gli allievi ti superano. Il giorno dopo ero nella Biblioteca del Seminario di Alessandria. Mi aspettava una serie di dieci volumi contenenti il commento di Giason del Maino al Corpus Iuris, stampato a Lione nel 1533 da Sebastian Gryphius ${ }^{1}$; uno solo di essi faceva eccezione, venendo da una edizione anch'essa lionese, del 1553, stampata da Guido Blasius (Prima super Codice) ${ }^{2}$, evidentemente aggiunta agli altri volumi in sostituzione di un volume perduto. Ma il dettaglio specifico sulle edizioni fu appurato dopo. Mentre mi preparavano a vedere i dati tipografici e a discuterli, cercavo di guardare attorno.

I volumi, forse anche grazie all'alluvione, non portavano veri piatti di legatura, e lasciavano in bella evidenza i fogli di guardia, variamente lacerati. I colori e la loro disposizione, così come i caratteri gotici a stampa e la loro distribuzione, già da qualche metro di distanza restringevano il campo a qualcosa di liturgico, un calendario. Ed evidentemente doveva trattarsi di un foglio di calendario non piegato, data la posizione 'rovesciata' di molti specchi di scrittura; ma le dimensioni non quadravano con le misure dei formati librari ordinari. Dunque un calendario-almanacco, una piccola cosa da portare con sé.

Così la scrittura Almanach, cui sottostavano alcune parole in caratteri ostentatamente latini, in contrasto con i gotici del resto dell'almanacco, arrivava come una conferma. Mi restavano due punti non chiari: perché in fogli non piegati? perché così tanti? Di norma chi lavora su frammenti assume un particolare babitus mentale di fronte al complicarsi delle ipotesi, forse determinato utilmente dalla complicazione stessa, per via di probabilità: è possibile che alcuni degli effetti di cui si cerca una causa, siano essi stessi una causa? Nello specifico la non piegatura spiega la quantità, e la quantità la non piegatura: materiali avanzati e non più utilizzabili, esattamente come oggi i calendari dell'anno precedente, che spesso si trovano ammassati nella carta gettata via. L'effetto evocativo non è piccolo o trascurabile, come in un'opera di Warhol, e certamente si pensa in modo vertiginoso a tutti gli anni come avrebbero potuto essere, a una molteplicità di realtà, molto lontano da quanto il buon senso vorrebbe.

(1) S. VON GÜLTLINGEN, Bibliographie des livres imprimés à Lyon au seizième siècle, V, Sébastien Gryphius, Baden-Baden \& Bouxwiller 1997 (Bibliotheca Bibliographica Aureliana, CLX; Répertoire bibliographique des livres imprimés en France au seizième siècle, Fascicule hors série), n. 218, p. 48 , in nove volumi, ove si indica che è una «réimpression de l'édition de 1530», ai nn. 118-126, con un solo esemplare completo, Cambridge St. John's College; variamente incompleti Gand, BU, Luxembourg, BN, Salamanca BU. A. PetTEgreE, M. WALsby, A. Wilkinson, FB. French Vernacular Books.
Books published in the French language before 1601, Leiden 2007, cit. in Universal Short Title Catalogue (www.ustc.ac.uk), dà un solo esemplare completo dell'ed. 1533. Notiamo, per precisione, che il volume super Infortiato è stampato da Jacques Myt.

(2) S. von GÜltLingen, Bibliographie des livres imprimés à Lyon au seizième siècle, V, Sébastien Gryphius, Baden-Baden \& Bouxwiller 2007 (Bibl. bil. Aureliana) n. 23, p. 29, un solo esemplare, Madrid, Bibl. Nac.; Universal Short Title Catalogue aggiunge Dresden, Sächsische Landesbibliothek e Konstanz, Heinrich-Suso-Gymnasium. 
Naturalmente appena l'occhio cadde sulla firma in nesso FRabelaesus, date le premesse (puramente casuali), non ci fu nessun senso di sorpresa. Nove copie, variamente conservate, dell'Almanach del 1535 di Rabelais si presentavano, associate ad un altro calendario (almeno così sembrava) certamente diverso; un solo volume, quello del 1553, non portava fogli di guardia. Insomma, all'interno dell'avara tradizione degli almanacchi di Rabelais, costituita da pochi frammenti, e anche da trascrizioni di testi scoperti, venerati e poi dispersi nuovamente, irrompevano ben nove copie, più o meno intere, dell'Almanach del 1535, del quale si conosceva, attraverso una trascrizione, il contenuto della Pronostication ${ }^{3}$. Avevo cognizione dell'interesse della scoperta, ma non esattamente la misura della rarità.

Naturalmente offersi il documento a chi senza dubbio ne sapeva più di me, ma alcuni mi incoraggiarono ad occuparmene personalmente, altri non trovandoci nulla di riportabile al 'carnevalesco' lasciarono perdere l'occasione. Presentai diverse volte il testo durante seminari, in particolare a Montréal, feci fare alcune riprese fotografiche professionali, sulla base delle quali mi trovai in grado di ricostruire sommariamente il calendario, integrando le parti mancanti in una copia con le parti leggibili in altre copie. Tutto questo avveniva prima che la Biblioteca del Seminario di Alessandria provvedesse al restauro dei volumi.

Il restauro fu fatto con intelligenza, senza separare i frammenti dell'Almanach dai testi di Giason del Maino: essi si trovano dove erano originariamente, non in cartelle a parte come si tende, improvvidamente, a fare. Come sempre accade nei restauri, quasi per definizione, alcuni elementi si guadagnano, altri si perdono: i fogli dell'Almanach sono perfettamente maneggiabili, e possono andare tranquillamente - salvo eventi dirompenti - incontro ad almeno un altro millennio, non presentando segni di acidificazione, muffe od altro agente di degenerazione in atto; la sostanziale cancellazione dei segni dell'incuria e della distratta conservazione fa viceversa perdere totalmente di vista il fatto che una testimonianza così importante non abbia sollevato alcun interesse nel quasi mezzo millennio che intercorre fra la loro fabbricazione e il loro ritrovamento. Un restauro virtuale, fatto attraverso trattamento di immagine da Graziella Pastore, mi ha comunque offerto un documento in grado di contemperare le diverse esigenze di recupero del dato originale senza perdite di dati accidentali e contingenti, ma storicamente rilevanti.

Diventa a questo punto importante provare a comprendere la storia materiale dei volumi che hanno accolto, legati a sé, gli Almanacchi di Rabelais. Quando si parla di fortuna o sfortuna critica e culturale di un autore si ha tendenza a cercarne testimonianza negli ambienti strettamente letterari, mentre si lasciano da parte altri ambienti ed aspetti importanti. È ragionevole immaginare che i dieci volumi di Giason del Maino, stampati a Lione, abbiano avuto una presenza e un uso in Italia nordoccidentale almeno per tutto il restante XVI secolo. Successivamente forse un qualche uso saltuario, che non ha lasciato traccia evidente nei volumi, ma in un'epoca in cui Giasone, famoso ed importante in Italia e in Francia come esponente dell'ultimo bartolismo, giurista e politico rilevantissimo, era ormai oggetto di indagine storica piuttosto che di uso corrente.

Ovviamente non esiste alcun legame altro che materiale e contingente tra Rabe-

(3) P.-P. PLAN, Bibliographie rabelaisienne. Les éditions de Rabelais de 1532 à 1711. Catalogue raisonné descriptif et figuré..., Paris 1904 dà, pp. 229. 232, in sintesi, per gli Almanacchi: 1533 citato ma non conservato (ed. Huchon, p. 936-937); 1535, citato ma non conservato (ed. Huchon, pp. 938-940);
1536 citato da Rabelais stesso, totalmente perduto; 1541, conservati due pezzi della pagina iniziale, tratti da due copie defl'Almanacco (ed. Huchon, pp. 941-950); 1546 menzionato, ma non se ne ha traccia; 1548 citato ma non conservato; 1550 menzionato ma non conservato; 1553 menzionato. 
lais, l'almanacco, Giason del Maino, e il fatto che Rabelais stesso citi Giasone come giurista nel Tiers livre:

Je ne seray hors de propos, si je ne vous raconte ce que dit Jo. André sus un canon de certain rescript papal addressé au Maire et Bourgeoys de la Rochelle: et aprés luy Panorme en ce mesme canon: Barbatia sur les Pandectes, et recentement Jason en ses conseilz, de Seigny Joan fol insigne de Paris, bisayeul de Caillette... ${ }^{4}$

e anche, brevemente, nel Pantagruel:

et tous les principaux Regens des Universitez, non seulement de France, mais aussi d'Angleterre et d'Italie, comme Jason, Philippe Dece, Petrus de Petronibus et un tas d' autres vieux Rabanistes 5 .

Altri elementi invece aiutano a comprendere una parte delle vicende dell'Almanacco, ed in particolare l'altro 'calendario' di cui si è detto. Precisiamo subito che non si tratta propriamente di un calendario, ma della parte iniziale di un Officio, che, come è normale, contiene un calendario; ma daremo successivamente i dettagli utili. I volumi del grande commentario in questione sono, in linea di massima, dotati di un foglio di guardia in inizio e di un foglio in fine, secondo il seguente schema, ove per brevità per Almanacco si intende l'Almanach di Rabelais, e con Officio il calendario anteposto all'Officio:

AN-3-IV-1.1: Prima pars super Digesto Veteri, con l'Almanacco in inizio e in fine

AN-3-IV-1.2: Secunda super Digesto Veteri, con Almanacco e Officio

AN-3-IV-2.1: Prima super Infortiato, con Officio e Officio

AN-3-IV-2.2: Secunda super Infortiato, con Officio e Officio

AN-3-IV-3.1: Prima super Codice, né Almanacco né Officio

AN-3-IV-4.2: Secunda super Codice, con Officio e Officio

AN-3-IV-15.1: Prima super Digesto Novo, con Almanacco e Almanacco

AN-3-IV-15.2: Secunda super Digesto Novo, con Almanacco e Almanacco

AN-3-IV-16: Repertorium in lecturas Iasonis, con Almanacco e Almanacco

Nel cercare di costituire una cronologia degli eventi dobbiamo fare alcune premesse. La prima riguarda l'edizione di Giason del Maino. Può sembrare incongruo, ma così è nei fatti, che un'opera monumentale come quella del commento, stampata a Lione nel 1533, sia divenuta rarissima. Nove volumi di dimensione notevole, circa 400x275 mm, portanti la stessa data (Lugduni, apud Sebastianum Gryphium, M.D.XXXIII), certamente destinati allo studio ed alla pratica, costituiscono il risultato di una impresa editoriale di grande impegno, cui dovette corrispondere un adeguato numero di copie: tuttavia, sulla base dei dati della Bibliotheca Bibliographica Aureliana citata disponiamo soltanto di una menzione indiretta (l'edizione del 1533 riprende l'edizione del 1530); l'esemplare completo, Cambridge, St. John's College, e un esemplare, Gent, Centrale Bibliotheek der Rijksuniversiteit, con la stessa lacuna presentata dalla nostra edizione. I non molti altri, quattro compreso il nostro, sono variamente incompleti; quanto al volume stampato successivamente (Lugduni, excudebat Blasius Guido, M.D.LIII), è stato evidentemente acquistato per compensare una lacuna determinatasi sin dall'inizio, o successivamente; notiamo che la stampa del 1553 dà soltanto la Prima e la Secunda super Codice, almeno a quanto ci resta: ci si

(4) Tiers livre, cap. 37, ed. M. HuCHON, Paris 1994, pp. 468-469.
(5) Pantagruel, cap. 10, ed. M. Huchon, Paris 1994, p. 251. 
può chiedere, senza avere una risposta altro che ipotetica, se la stampa presso Guido Blasius, di buona qualità, ma di stampatore di minor peso, non sia nata per compensare carenze di tiratura dell'edizione del 1530 e forse del 1533.

Per quanto è del nostro esemplare, che possiede la Secunda super Codice, ma non la Prima, notiamo subito che il volume del 1553 non ha gli stessi fogli di guardia degli altri volumi, e inoltre non porta sul frontespizio traccia dei nomi dei precedenti possessori che figurano invece nei volumi del 1533, in genitivo Francisci Rinaldi, e nel solo Repertorium anche Anthonii Alamandi, tutti e due biffati, senza che si possa stabilire quale sia stato il primo dei due a possedere i volumi. Precisiamo, nessun segno di appartenenza ad enti religiosi antecedente al vistoso timbro di possesso "Libreria - Seminario vescovile - Alessandria”, databile agli ultimi decenni del XIX secolo.

Non mi è per ora stato possibile identificare i possessori, che non figurano né nella mia base dati, né in altre cui ho avuto accesso; ringrazio collettivamente tutti i bibliotecari che gentilmente mi hanno offerto i loro dati. Tuttavia, senza con questo voler trarre certezze assolute, noto che i cognomi Rinaldi, Rinaudo, Rinaudi, Allamandi, Allemandi sono oggi ben diffusi in Piemonte, in particolare in area sudoccidentale (Torino, Asti e dintorni) con concentrazioni grosso modo coincidenti ${ }^{6}$, mentre per la Francia il tipo Allemand e il tipo Renaud hanno concentrazione di distribuzione non coincidente (Sudest il primo, Ovest il secondo). Posto che queste note di possesso non figurano nel volume del 1553, potremmo desumere che in data precedente i volumi si trovassero in Italia, in un intervallo che possiamo immaginare, per ora, tra il 1533 e il 1553 . Tutto questo non ha nulla di sorprendente, posto che l'area piemontese compra moltissimo della produzione lionese; al tempo stesso, nel periodo in cui l'area piemontese è di dominio francese (1536-1562) la distinzione diviene ancora più tenue. Senza voler avere certezze assolute, possiamo ritenere che i volumi si trovassero in area cisalpina in quell'intervallo di tempo.

Un secondo elemento di datazione ci arriva dall'altro "calendario". Esso ha una natura del tutto differente, anch'essa non priva di contraddizioni. Nel frammento di calendario/officio annesso al volume AN-3-IV-4.2, Secunda super Codice, si ha un frontespizio: Ordo Divini Officii / Quisquis es: qui sacrum Deo Optimo Trino et Uno Sermonem / quod divinum officium appellant / Ex more Romane curie impen/dere cupis: hos perlege et imitare Canones: Quos Eustachius / La Porta Sanctissime Trinitatis Metropolitane Taurinensis / Ecclesie Concanonicus tibi non minore cura quam ingenio concinalvit: Exolves enim (mibi crede) munus boc tuum ad annum / binc sextum et aere et labore parvo: Atque omnem erroris nelbulam prorsus abs te fugabis. Vale

$\mathrm{e}$ in basso:

Venundantur Taurini in officina / Antonii Galini de Verrolengo ingabis.

Notiamo che nel titolo dedicatorio, all'ultima riga fugabis sembra corretto su

Non constano altre copie di questo Officio, e di conseguenza non ci si può basare su dati diversi da quelli fornitici da questo esemplare incompletissimo. Prima di tutto si osserva una caratteristica non unica, ma certo non frequentissima: anziché anteporre all'Officio un calendario perpetuo, il curatore ha prefertito seguire una linea che gli deve essere parsa lungimirante, ma non ha sortito effetto. Invece del consueto calendario perpetuo ha preferito mettere una serie di calendari di singoli anni; abbiamo, nella parte che si è conservata, gli anni dal 1530 (dicembre) al 1536,

(6) In sintesi si possono usare i siti www.gens. info/italia/ e, per la Francia, www.geopatronyme. com. I dati che si possono trarre da queste fonti hanno diversa consistenza storica. Di norma, quan- do sono di buon numero e di buona compattezza, come nel nostro caso, possono dare indicazioni di buona attendibilità. 
la qual cosa coincide con quanto scritto nel titolo "ad annum binc sextum"; avremmo dunque, salvo qualche lacuna e qualche ripetizione, il calendario intero, su sei anni, per il quale il compilatore-imprenditore promette un uso "aere et labore parvo": poca spesa, della quale non sappiamo, e poca fatica, perché effettivamente i calendari perpetui chiedono per il loro impiego un impegno attento.

L'editore sperava di stampare un Officio con un calendario tale che il settimo anno divenisse necessario comprare un nuovo Officio (evidentemente anch'esso implicante poca spesa e poca fatica). Naturalmente deve aver sottovalutato il fatto che sì il settimo anno sarebbe stato necessario comprarne un altro, ma man mano l'Officio diventava meno interessante e più difficilmente commerciabile. In prossimità della sua scadenza diventava del tutto inutile, e dunque poteva seguire la stessa sorte dell'Almanacco di Rabelais del 1535.

Posto che l'operazione di annessione dei fogli di guardia è avvenuta evidentemente nello stesso momento, come dimostra il volume AN-3-IV-1.2, che contiene sia l'Almanacco sia l'Officio, dobbiamo immaginare che questo momento fosse verso il 1536, con tutti e due i documenti ormai privi di utilità. Che nel lavoro di annessione dei fogli di guardia abbia avuto un qualche ruolo Rabelais stesso mi pare poco congruo, non essendo plausibile che disponesse di copie ancora non piegate dell'Officio.

D'altra parte l'Officio aveva una sicura destinazione piemontese, come mostra con chiarezza l'indicazione Venundantur Taurini in officina / Antonii Galini de Verrolengo, e come confermano alcune presenze caratteristiche nel calendario, come Massimo di Torino il 25 giugno, in rosso; san Secondo di Torino il 24 ottobre; i santi Solutore e Avventore il 20 novembre (nel calendario del 1533).

L'Officio, destinato con certezza dunque all'area torinese, non è necessariamente stampato a Torino; pur non essendo un dato irrefutabile, l'indicazione che l'Officio è in vendita a Torino nell'officina di Antonio Galini fa pensare che l'officina non l'abbia prodotto, ma lo venda soltanto, e se fosse stato prodotto in una tipografia torinese, essa stessa avrebbe potuto venderlo.

Di fronte ad una doppia possibilità, che i fogli dei calendari siano stati apposti in Italia, e nello specifico a Torino (o nella diocesi di Torino), oppure a Lione prima di essere esportati, è piuttosto questa seconda che pare essere più probabile. Nel primo caso dovremmo pensare che in un determinato anno, che potremmo immaginare essere il 1536, in una bottega di rilegatore di Torino si trovassero i volumi di Giasone stampati a Lione tre anni prima (già legati o a fogli sciolti non importa), un Officio torinese ormai non più vendibile, ma ancora non piegato e non legato, e un calendario lionese non più utilizzabile, ma ancora non piegato e non legato. Nel secondo caso dovremmo pensare ad una bottega lionese ove si trovassero i volumi lionesi di Giasone, le copie di almanacco di Rabelais non vendute, e qualche copia di Officio destinato alla diocesi di Torino, ma prodotto a Lione. Questa seconda possibilità parrebbe più plausibile.

Un ulteriore elemento può contribuire a sostenere questa seconda possibilità. L'Almanacco di Rabelais non porta filigrana, mentre l'Officio ne porta diverse (colonna, croce in goccia), nessuna delle quali strettamente comparabile con filigrane registrate da Briquet (ma siamo già a metà del XVI secolo, quando i rilevamenti di Briquet si diradano), ma tutte riferibili a tipi esistenti. Le filigrane dell'Officio sono in taluni casi identiche alle carte di guardia bianche dei volumi; non hanno viceversa mai somiglianza con quelle della carta su cui sono stampati i volumi di Giason del Maino. Se esistono questa identità e questa distinzione, se ne dovrebbe trarre che la produzione dell'Officio e la legatura dei volumi è avvenuta in una tipografia che usava la stessa carta per stampare e per legare, quando fosse il caso. Tutto questo comporta qualche altra considerazione.

La non coincidenza delle filigrane dei volumi e dei fogli di guardia non pone 
problemi, dato che comunque fra la data di stampa (1533) e la data di annessione dei fogli di guardia (che ragionevolmente porremmo nel 1536, pochi mesi più, pochi mesi meno) è intercorso tempo. Viceversa difficilmente penseremo alla tipografia di Gryphius come luogo della legatura: le filigrane dell'Officio non datano a dopo la fine del 1530 (data di inizio del calendario), e i sei anni di distanza dalla legatura sembrano un po' troppi per immaginare che una partita di carta restasse inutilizzata in una tipografia così importante per un tempo così lungo. Dovremmo in conclusione pensare che i volumi, prodotti a Lione, siano stati legati in Lione stessa alcuni anni dopo, al momento di una vendita, e probabilmente nella stessa tipografia, più piccola e non ancora identificata, che ha prodotto l'Officio, destinato a Torino. Se la tipografia in questione fosse la stessa che ha prodotto anche l'Almanacco, vale a dire Juste, non abbiamo modo di sapere, e non paiono esser disponibili ulteriori fonti.

I volumi, immediatamente o no, passarono poi nelle mani dei due possessori Franciscus Rinaldi e Anthonius Alemandi. Certamente essi videro l'Almanacco, ma non ci fecero gran caso: su diversi fogli dell'Almanacco e democraticamente dell'Officio ci sono sia calcoli, sia rinvii a passi del testo di Giasone.

La cronologia si assesterebbe dunque secondo la seguente successione: 1530 stampa dell'Officio; 1533 stampa del testo di Giasone, forse ad eccezione del volume sostituito; non è escluso infatti che sin dall'inizio mancasse il volume della Prima super Codice; 1535 stampa del calendario; 1536 o poco dopo legatura definitiva dei volumi; i volumi passano immediatamente, o comunque poco dopo, ai due possessori, in una successione che non sono in grado di stabilire; forse i volumi vengono ceduti ad altro possessore; nel 1553 o dopo, si aggiunge il volume mancante, che non porta nota di possesso, o perché i volumi erano tutti ormai passati ad altro proprietario non abituato a mettere la sua nota di possesso, oppure perché erano ancora in mano dello stesso proprietario, che non ha apposto la sua firma sul frontespizio. Questa seconda ipotesi non è peregrina: le precedenti note di possesso sembrano essere state apposte in una unica operazione, non ad una ad una; l'arrivo di un nuovo volume non necessariamente avrebbe determinato la ripresa dell'operazione.

Sin qui le vicende esteriori e materiali, non prive di importanza perché ci mostrano la presenza di questo materiale in Italia, e la sua nascita a Lione; e ci mostrano un sostanziale disinteresse per l'attribuzione del calendario a Rabelais, al contrario di quanto è accaduto per l'Almanacco del 1541, che ci giunge in due esemplari lacunosi, sui quali in modo chiarissimo disse Charles Marty Laveaux, opportunamente ripreso da F. Moreau nell'ed. Huchon ${ }^{7}$ :

On s'explique d'ailleurs fort bien que les autres parties de cet opuscule n'aient pas été recueillies: si après 1541 quelques exemplaires invendus ont servi à garnir les reliures, les amateurs n'ont remarqué et mis de côté que la feuille qui contenait le titre et par conséquent le nom de Rabelais; le reste a passé inaperçu.

Il confronto fra i due modi di conservare il testo (che nelle riproduzioni allegate all'ed. Huchon non sono disposti nella caratteristica forma determinata dall'imposizione), l'uno legato alla conoscenza di Rabelais, l'altro a un sostanziale disinteresse, sembrano marcare due distinte condizioni areali di fortuna dell'autore. Per quanto riguarda i due frammenti descritti da C. Marty Laveaux non sappiamo molto oltre al fatto che esistono: uno fu acquistato dalla Biblioteca già in forma di frammento,

(7) Nelle Notes sur le texte, nell'ed. delle Euvres complètes, p. 1715; il passo di C. MARTy LAVEAuX, ripreso anche da Plan, è in Les Euvres de maistre
François Rabelais, Paris, Lemerre, 1868-1903, IV, p. 360 . 
l'altro fu scoperto da Marty Laveaux fra «débris informes que j'avais été chargé de classer»: come spesso accade, una volta interrotta la contiguità fra i frammenti di legatura e libro, tutte le informazioni storico-culturali relative al frammento vanno perse. Non abbiamo modo di sapere come, dove e quando i frammenti sono stati distaccati e ritagliati. La testimonianza si è trasformata in reliquia ${ }^{8}$.

Molto in breve diamo alcuni ragguagli di contenuto, in particolare per quanto non è dato nei frammenti precedentemente noti, e rinviando per il testo all'edizione facsimilare di prossima pubblicazione presso l'editore Garnier.

Prima di tutto l'indicazione del libraio, che si trova nell'ultima pagina; il colore rosso è rappresentato dal corsivo: "On les vend ches François Juste / En la rue Mercière devant / Nostre Dame de / Confort a / Lyon”. Non è fuori luogo pensare che il venditore fosse anche lo stampatore, posto che Juste stampò molte volte diverse opere di Rabelais, nell'ordine cronologico: 1532 Pantagrueline prognostication'; 1533 Pantagruel $^{10} ; 1533$ forse una Pantagrueline prognostication ${ }^{11} ; 1534$ Pantagruel $^{12} ; 1534$ Pantagrueline prognostication ${ }^{13} ; 1535$ Gargantua $^{14} ; 1537$ Gargantua $^{15} ; 1537$ Pantagruel e Pantagrueline prognostication ${ }^{16} ; 1542$ Gargantua $^{17} ; 1542$ Pantagruel $^{18}$; ad esse ovviamente si aggiunge il nostro Almanacco, e forse anche altri, come quello del 1541. Nel complesso i numeri sono molto piccoli; su questo è opportuno il richiamo alle belle pagine introduttive della Huchon ${ }^{19}$. I piccoli numeri, così pericolosamente prossimi allo zero, paradossalmente fanno prospettare come importanti e forse innovative tutte le prossime scoperte di materiale rabelaisiano.

Poco sotto l'indicazione della data, 1535, in caratteri ebraici e greci, un piccolo pezzo di bravura, e la firma FRabelaesus di cui si è detto.

Ciascun blocco di testo contiene metà mese, e con l'opportuno taglio e l'opportuna piegatura del foglio, giunge a costituire due fascicoletti oblunghi di circa 90x70 $\mathrm{mm}$, che probabilmente dovevano essere cuciti assieme. Alcune parti infatti mantengono la loro utilità nel corso dell'anno: l'indicazione delle date di riferimento; la lettera dominicale $\mathrm{C}$ (che caratterizzerà tutte le domeniche del calendario), il numero

(8) Notiamo che le diverse riproduzioni fotografiche (Huchon, Plan, Marty Laveaux) orientano le immagini di ciascun riquadro di calendario in modo da favorire la lettura, ma non rendono conto della giacitura dei frammenti, che nell'originale sono disposti secondo quanto la tecnica dell'imposizione vuole; fedele all'originale invece S. Rawles-M. A. Screech, A New Rabelais Bibliography, Genève 1987 («Travaux d' Humanisme et Renaissance», 219) [d' ora in poi RAWLES-SCREECH], 97, pp. 503-506.

(9) RaWLes-SCREeCH, 14, pp. 113-116, tre copie conservate.

(10) RAWles-Screech, 7, pp. 84-88, una sola copia conosciuta, distrutta durante la guerra.

(11) RAWles-SCREECH, 16, pp. 120-123, nessuna copia conservata.

(12) RAWLES-SCREECH, 8, pp. 88-91, quattro copie conservate.

(13) Rawles-Screech, 18, pp. 126-128 quattro copie conservate

(14) RAWLES-SCREECH, 19, pp. 129-131, una copia conservata; ancora nel 1535, RAWLES-SCREECH, 20, pp. 132-138, quattro copie.

(15) RAWLES-SCREECH, 22, pp. 142-144, quattro copie, e altre perdute.

(16) RAWLES-SCREECH, 11, pp. 101-103, una copia conservata, altre due segnalate.

(17) RAWLES-SCREECH, 23, pp. 145-147, sette copie.

(18) RaWles-Screech, 12, pp. 104-108, almeno tredici copie. Cfr. anche S. von GültLINGEN, Bibliographie des livres imprimés à Lyon au sezième siècle, IV, Baden-Baden, Bouxwiller 1996 (Bibliotheca bibliographica Aureliana, CLIV, Répertoire bibliographique des livres imprimés en France au seizième siècle); le copie recensite dalla Gültlingen sono in numero minore rispetto al repertorio di Rawles e Screech, ma è comunque utile estrarne $i$ dati, comparabili con altri all'interno della stampa lionese nel suo assieme. Il prospetto delle opere di Rabelais pubblicate da Juste è il seguente, ed è particolarmente rilevante la rarità delle copie superstiti ivi censite (indico le pagine del repertorio della Gültlingen e il numero): 1532 Pantagrueline, p. 201 n. 7; 1533 Pantagruel, p. 203 n. 14 una copia; 1534 Pantagruel, p. 205 n. 22 due copie; 1534 Pantagrueline, p. 205 n. 23 due copie; 1535 Gargantua, p. 207 due copie; 1537 Gargantua, p. 214 n. 69 due copie; 1537 Pantagruel + Pantagrueline, p. 215, n. 70 una copia; 1542 Gargantua, p. 219, n. 90 tre copie; 1542 Pantagruel, p. 219, n. 91, quattro copie.

(19) M. Huchon, Rabelais, Paris 2011, in particolare le pp. 12-13. 
aureo XVI (che indica la posizione dell'anno nel ciclo diciannovenale di Dionigi il Piccolo), l'indizione romana VIII (posizione nel ciclo indizionale di quindici anni); la distanza tra Natale e la Domenica Grassa (la domenica precedente il mercoledì delle Ceneri, la Settuagesima, Brandons (prima domenica di Quaresima), Pasqua, Rogazioni, Ascensione, Pentecoste, inzio dell'Avvento. Successivamente: Congiunzioni della Luna con Marte, Saturno e la coda del Dragone; la spiegazione dei segni zodiacali; le abbreviazioni usate per i tempi della luna, la numerazione delle domeniche dopo Pentecoste. Poi i simboli delle fasi lunari, le indicazioni di "mattino, pomeriggio", i simboli per indicare i tempi opportuni o non opportuni per l'assunzione di medicine, salassi, ventose e bagni, piantare e seminare, slattare i bambini, tagliare le unghie, la luna impedita. Le date delle fiere di Lione.

In fine al mese di febbraio, dove avanza spazio, ulteriori legende: durata del giorno, inizio e fine delle fiere di Lione.

Questa parte, e vedremo anche l'altra, corrisponde in modo alquanto compiuto a quanto ci è rimasto dell'Almanacco del 1541, con piccole varianti. Non essendoci pervenuto l'elenco delle fiere di Lione del 1541, dobbiamo accontentarci di ritrovarne traccia nella parte calendariale, p. es. il 3 novembre.

L'Almanacco è ordinato in colonne, nello stesso modo che si riscontra nell'Almanacco del 1541, nell'ordine: il giorno del mese; i giorni della settimana (prima di essi, in rosso i numeri delle domeniche dopo la Pentecoste, ove opportuno), il nome del santo del giorno, o della ricorrenza, il segno zodiacale in cui entra la luna, l'età della luna, le sue ore, seguite da tutti i segni corrispondenti alle funzioni sopra elencate.

L'esattezza dei dati astronomici è un problema complesso che richiede una pratica consolidata. Per questo, al di là di quelle che possono oggi essere le credenze personali, mi sono rivolto ad una nota astrologa, Grazia Mirti, che è in grado di eseguire i calcoli con esattezza, e di capirne l'esattezza di computo e la pertinenza, anche tenendo conto della latitudine e della longitudine di Lione; la ringrazio molto per la gentilezza e per la competenza in questo campo complesso, offerta con grande rispetto per i diversi punti di vista. Fatti salvi pochissimi scarti sull'età della luna - cosa che dà talora minutissimi scarti, essendo approssimata all'unità - i conti sembrano essere esatti. Possiamo su questo dire che l'Almanacco è un prodotto nel quale Rabelais, almeno nella parte esaminata, ha lavorato con puntiglio. La sua opera assomiglia certamente più a un almanacco qualsiasi che alle opere maggiori di Rabelais.

Lo studio sulla scelta dei santi da mettere in calendario è ovviamente subordinata all'edizione del testo, per la quale rinviamo alla citata edizione con facsimile di prossima pubblicazione presso Garnier. Per ora tuttavia è possibile trarre alcune indicazioni di ordine generale.

La prima è che non è bene dare per definitivamente acquisito il patrimonio delle cose note: le nostre nove copie lo dimostrano. Paradossalmente questo Rabelais abbandonato con sostanziale noncuranza finisce per essere, forse, l'opera che ci giunge, nel XVI secolo, nel maggior numero di copie. Analogamente, in secondo luogo, le ricognizioni anche casuali possono far riemergere ancora altro, soprattutto se teniamo conto che tutte le opere giunteci in un solo esemplare erano opere non lontane dalla perdita totale. Non è imprudente pensare che qualche altro caso possa presentarsi, e proprio cercando fra i materiali di scarto aumentano le probabilità di trovare ciò che si è perso. Una ricognizione di ampio respiro (FRAGMENTA-FRAGMENTS) sui frammenti contenuti in legature d'archivio del Piemonte sta dando frutti di notevole qualità e quantità (in questo momento attorno ai 1400 frammenti, destinati a triplicarsi nel tempo della ricerca, ed in prospettiva a decuplicarsi), e già di fronte a questo numero di frammenti sta rivelando la sua esistenza una sorta di genere storico-letterario, costituito da testi soggetti a un deperimento particolarmente intenso e rapido. I dati quantitativi e statistici per ora non sono stati elaborati, ma le stesse 
ragioni che hanno fatto disperdere tante edizioni di Rabelais, sono quelle che hanno trasformato in materiale di legatura le tante migliaia di manoscritti che stiamo man mano scoprendo. La circostanza è particolarmente favorevole, sul piano quantitativo. Che poi riemergano altre cose di Rabelais o di altri, questo è del tutto imprevedibile, ma sembra non inopportuno sperarlo.

ALESSANDRO VITALE-BROVARONE 